\title{
Assessment of Knowledge, Behavior and Attitude of School Children towards Smoking
}

Kristina Tot Veres ${ }^{1^{*}}$, Biljana Zvezdin ${ }^{2}$ and Danijela Vukosav ${ }^{2}$

${ }^{1}$ Military Medical Center, Novi Sad, Serbia

${ }^{2}$ The Institute for Pulmonary Diseases of Vojvodina, Novi Sad, Serbia

\begin{abstract}
Background: Tobacco use is one of the greatest threats for public health. The future prosperity of the tobacco industry depends directly on the youth's tendency to experiment. AIMS: The aim of the study was to examine the prevalence of cigarette smoking and the tobacco consumption among schoolchildren between age 11 and 15 , to examine the knowledge regarding what harmful effects tobacco smoke has on health and to assess the extent of exposure to tobacco smoke in the home environment.
\end{abstract}

Study design/Methods: The testing is a cross-sectional study conducted on students of upper grade levels in primary school "Miloje Čiplić" in Novi Bečej, Serbia in 2012. A total of 220 schoolchildren were tested. Upon verbal instructions they filled in a questionnaire containing 24 questions, designed after the model by a joint survey of the Center for Disease Control and Prevention and the World Health Organization.

Results: Of all respondents, $35 \%$ of students have already smoked their first cigarette, and $5.9 \%$ of them were everyday smokers. Thirty-two point seven percent of the children came from families where both the mother and the father were non-smokers, $24.1 \%$ of the them had both their parents smoking, $16.4 \%$ came from homes where the father was the only smoker and $20 \%$ where the mother was the only smoker. In $3.6 \%$ of cases someone else in the family was the smoker. $56.7 \%$ of the children were exposed to smoking in their homes and $74.5 \%$ of students were occasionally exposed to tobacco smoke because their guests were allowed to smoke. $25.5 \%$ of the students thought smoking was a disease. $44.5 \%$ of schoolchildren knew what secondhand smoking was, while $17.7 \%$ of students thought that it meant smoking in company of their friends, $6.4 \%$ thought it was occasional smoking. Of all children $27.3 \%$ stated they did not know what secondhand smoking was.

Conclusion: In this study it has been shown that a large percentage of surveyed schoolchildren between 11 and 15 years old have tried cigarettes, and the number of daily smokers is worryingly high.

Keywords: Schoolchildren; Smoking; Youth; Secondhand smoking; Peer smoking

Abbreviations: CDC: Disease Control and Prevention; WHO: World Health Organization; GYTS: Global Youth Tobacco Survey

\section{Introduction}

Tobacco use is one of the greatest threats for public health. It is assumed that in this century smoking will cause the early deaths of 1000 million people [1]. Despite the grim statistics millions of lives can be saved by preventing schoolchildren from experimenting with cigarettes and subsequently developing a nicotine addiction. In the European Union $19.8 \%$ of young people between age 13 and 15 are smokers and one of every three non-smokers in the same age group intends to try smoking in the following year [2]. The future prosperity of the tobacco industry depends directly on the youth's tendency to experiment [3]. There are a few different ways to help decrease the consumption of tobacco among the youth: by decreasing the proportion of schoolchildren who will try out smoking cigarettes or by increasing the number of schoolchildren who will stop smoking. Within big social communities these strategies can be realized by the help of campaigns conducted by public information offices or national programs that can control the use of tobacco; whereas, within smaller social communities the same form of educational support can be provided to families, schools and to the health care institutions; finally, prices of cigarettes can be raised by legislative measures, taxes can be increased on tobacco or regulations can be made stricter to prevent young people from having access to tobacco [4]. The main principles that need to be followed when putting the above mentioned changes in effect are: restriction which involves the increase of prices of tobacco products, the prohibition of selling tobacco to juveniles and ensuring smoke-free areas; education for children in schools and for the families, utilising the tools of media as well; obstruction of selling tobacco products by prohibition of advertising the same, launching promotional campaigns and putting graphic warning labels on the tobacco products. These activities are intended to raise awareness of the harmful effects of smoking and to represent smoking as a socially unacceptable behaviour [5].

The aim of this research is to observe the frequency of smoking among schoolchildren between age 11 and 15, to assess the extent of knowledge concerning what harmful effects the tobacco smoke has on health and to examine the extent of exposure to tobacco smoke in the homes of the schoolchildren participating in the test.

\section{Material and Methods}

This research is a cross-sectional study on students of higher grade levels in the Primary School of "Miloje Čiplić" in Novi Bečej, Serbia in 2012. In total 220 students were tested of which 139 attended classes

*Corresponding author: Kristina Tot Veres, Military Medical Center, Novi Sad, Milana Stanivukovića 17/18, 23000 Zrenjanin, Serbia, Tel: 023-532356, 064-155-60-49; E-mail: krisz@sbb.rs

Received October 27, 2015; Accepted December 11, 2015; Published December 18,2015

Citation: Veres KT, Zvezdin B, Vukosav D (2015) Assessment of Knowledge Behavior and Attitude of School Children towards Smoking. J Pulm Respir Med 5: 297. doi:10.4172/2161-105X.1000297

Copyright: @ 2015 Veres KT, et al. This is an open-access article distributed under the terms of the Creative Commons Attribution License, which permits unrestricted use, distribution, and reproduction in any medium, provided the original author and source are credited. 
in the Serbian language and 81 in the Hungarian language. In the presence of their class teachers after receiving some verbal instructions the children completed the questionnaire that consisted of 24 questions (Supplementary file) designed after the common questionnaire of Center for Disease Control and Prevention (CDC) and the World Health Organization (WHO). All students of the study voluntarily agreed to participate in the test. There were no incorrectly filled out questionnaires. The children filled out the questionnaire by themselves and anonymously. In this study, smoking is defined as smoking cigarettes.

Data was processed with the use of personal computer applying statistical programs of SPSS Windows and MS Excel. Standard statistical methods were used to determine differences between each statistical groups (t-test, $\mathrm{x}^{2}$ test). Results are presented in charts and column diagrams.

\section{Results}

This survey was undertaken on a total of 220 students between the fifth and eight grades in the Primary School of "Miloje Čiplić". The distribution of children in respect of their grade levels, gender and the language of teaching is shown in Table 1 and the percentage ratio is shown in Figure 1.

Of all interviewees 35\% (77) never tried smoking and 5.9\% (13) smoked every day. The distribution of the number of the children who tried smoking before and who were everyday smokers is shown in Table 2

Table 3 indicates that children of all grades commonly smoke less than 5 cigarettes a week. Chi-square test showed that the number of students who were smoking is not statistically correlated to which grade they were in $\left(\chi^{2}=11.54, \mathrm{df}=15, \mathrm{p}=0.173\right)$.

To the question what smoking was, $71.4 \%$ (157) of the children answered that it was a bad habit, $25.5 \%$ (56) thought it was a disease, 9.5\% (21) believed it was a rude behavior, $6.4 \%$ (14) equated smoking with pleasure and $3.2 \%$ (7) thought that it was simply a fun.

Smoking has a negative impact on health, $89.1 \%$ (196) of the children agreed with this statement, however, 5\% (11) did not know what effects it had on health.

Smoking causes addiction, 65.9\% (145) of the children believed this to be true, the same number of children stated that a smoker cannot quit smoking easily, $10 \%$ (22) thought that smoking had

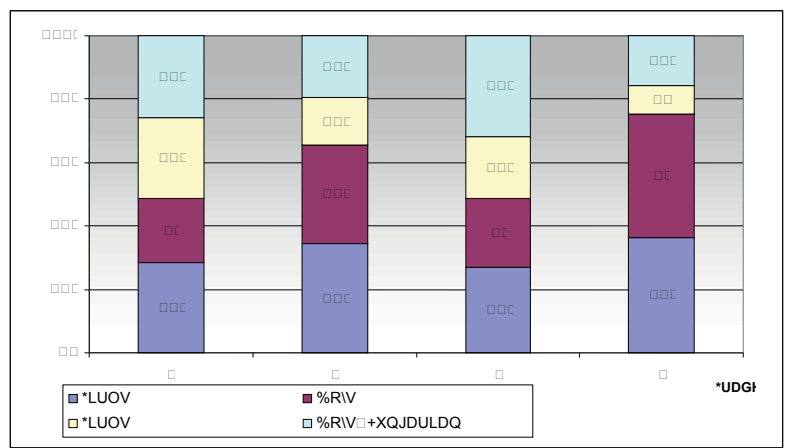

Figure 1: Distribution of pupils in respect of their grade levels, genders and the language of teaching. no effect on health and $17 \%$ (39) did not know what effects it had on health. Quitting smoking is easy, $17.7 \%$ (39) of the interviewee chose this statement.

Tables 4 and 5 shows the distribution of responses to the question whether tobacco smoke harms the environment or only the smoker in respect of the grade levels and gender. Chi-square test showed that different classes and genders do not significantly differ in their opinions on the question $\left(\chi^{2}=7: 46, d f=9, p=0.589,\left(\chi^{2}=2.89, d f=3, p=0.408\right)\right.$.

Table 6 represents the distribution of responses to the question whether tobacco smoke harms the environment or only the smoker in respect of the nationalities of the children. Chi-square test showed that students of Serbian and Hungarian classes differ significantly on this subject $\left(\chi^{2}=8.68, \mathrm{df}=3, \mathrm{p}=0.034\right)$.

Children of the $6^{\text {th }}, 7^{\text {th }}$ and $8^{\text {th }}$ grade believed that second-hand smoking meant staying in a smokey room, while a large number of schoolchildren in the 5th grade indicated that they did not know. Chisquare test showed that the children of the $5^{\text {th }}, 6^{\text {th }}, 7^{\text {th }}$ and $8^{\text {th }}$ grades were on a different opinion in a significant way about what they thought second-hand smoking was $\left(\chi^{2}=27.22, d f=12, p=0.007\right)$ (Table 7).

Table 8 shows that schoolchildren of different sexes differ statistically in a significant way in respect of what they think constitutes second-hand smoking $\left(\chi^{2}=9.34, \mathrm{df}=4, \mathrm{p}=0.051\right)$. Both girls and boys believed that second-hand smoking was equal to staying in a smokey room, but it is worth noting that some children chose to state "I have never thought about it". Up to $14.3 \%$ of the boys reported that they were not thinking about it, and only $2.9 \%$ of the girls.

Table 9 represents that schoolchildren of Hungarian and Serbian classes differ significantly in their opinions regarding what constitutes second-hand smoking $\left(\chi^{2}=64.64, \mathrm{df}=4, \mathrm{p}=0.000\right)$. Most of the children in the Hungarian departments wrote they did not know, and most of the children in the Serbian department wrote that second-hand smoking was staying in a smokey room.

Thirty-two point seven percent (72) of the children came from families where both the mother and the father were non-smokers, $24.1 \%$ (53) of the them had both their parents smoking, $16.4 \%$ (36) came from homes where the father was the only smoker and 20\% (44) where the mother was the only smoker. In $3.6 \%$ of cases someone else in the family was the smoker.

Children in whose home the guests were allowed to smoke tried out cigarettes in greater proportion than the children whose guests were not permitted to smoke. Up to $76 \%$ of children whose guests were not allowed to smoke in their homes have never tried cigarettes. The difference is statistically significant here $\left(\chi^{2}=6.64, \mathrm{df}=2, \mathrm{p}=0.036\right)$ (Table $10)$.

Table 11 shows that the children whose peers smoked in their presence tried smoking in greater proportion than the children whose peers were not smoking in their presence $\left(\chi^{2}=28.18, d f=2, p=0.000\right)$. About $50 \%$ of the students whose peers smoked in their presence tried smoking before, while $88.5 \%$ of the students in whose presence their peers never smoked have never tried smoking.

\section{Discussion}

A global survey was carried out by Global Youth Tobacco Survey (GYTS) in years 2003 and 2009 and it included Serbia as well [6]. This research was carried out in order to explore the use of tobacco products and the accessibility to the same, exposure to second-hand smoking and to advertisements of the tobacco products, quitting smoking and 
Citation: Veres KT, Zvezdin B, Vukosav D (2015) Assessment of Knowledge, Behavior and Attitude of School Children towards Smoking. J Pulm Respir Med 5: 297. doi:10.4172/2161-105X.1000297

Page 3 of 7

\begin{tabular}{|c|c|c|c|c|}
\hline Grades & $\begin{array}{c}\text { Girls } \\
\text { (Serbian class) }\end{array}$ & $\begin{array}{c}\text { Boys } \\
\text { (Serbian class) }\end{array}$ & $\begin{array}{c}\text { Girls } \\
\text { (Hungarian class) }\end{array}$ \\
\hline 5 th & 10 & 7 & 9 \\
\hline 6 th & 23 & 21 & 10 \\
\hline 7th & 11 & 9 & 8 \\
\hline 8th & 28 & 30 & 7 \\
\hline \multirow{2}{*}{ TOTAL } & 72 & 67 & 13 \\
\hline
\end{tabular}

Table 1: Distribution of children in respect of their grade levels, gender and the language of teaching.

\begin{tabular}{|c|c|c|c|c|c|c|c|c|}
\hline \multirow[b]{2}{*}{ Grades } & \multicolumn{4}{|c|}{ Tried smoking } & \multicolumn{4}{|c|}{ Everyday smokers } \\
\hline & Girls & Boys & Total & $\%$ & Girls & Boys & Total & $\%$ \\
\hline 5th & 4 & 2 & 6 & 17,1 & 0 & 0 & 0 & 0 \\
\hline 6th & 3 & 7 & 10 & 14,9 & 0 & 0 & 0 & 0 \\
\hline 7th & 7 & 12 & 19 & 46,3 & 1 & 3 & 4 & 9,8 \\
\hline 8th & 21 & 21 & 42 & 54,5 & 2 & 7 & 9 & 11,7 \\
\hline Total & $35^{*}$ & $42^{*}$ & 77 & 35,0 & $3^{* *}$ & $10^{* *}$ & 13 & 5,9 \\
\hline \multicolumn{9}{|c|}{$\begin{array}{ll}*{ }^{*} x^{2}=0,37 & p>0,05 \\
{ }^{* *} x^{2}=3,50 & p>0,05\end{array}$} \\
\hline
\end{tabular}

Table 2: Distribution of children who have tried smoking before in respect of their grade levels and gender.

\begin{tabular}{|c|c|c|c|c|c|c|c|c|c|c|c|}
\hline & & & \multicolumn{8}{|c|}{ How many cigarettes do you smoke per week } & \multirow[b]{2}{*}{ Total } \\
\hline & & & 5 cigarettes & $\begin{array}{l}10 \\
\text { cigarettes }\end{array}$ & 15 cigarettes & $\begin{array}{l}20 \\
\text { cigarettes }\end{array}$ & $\begin{array}{l}\text { More than } \\
\text { cigarettes }\end{array}$ & 20 & $\begin{array}{l}\text { Less than } \\
\text { cigarettes }\end{array}$ & & \\
\hline \multirow{4}{*}{ Class } & 5 & $\begin{array}{l}\text { Total } \\
\%\end{array}$ & $\begin{array}{l}0 \\
0,0 \%\end{array}$ & $\begin{array}{l}0 \\
0,0 \%\end{array}$ & $\begin{array}{l}0 \\
0,0 \%\end{array}$ & $\begin{array}{l}0 \\
0,0 \%\end{array}$ & $\begin{array}{l}0 \\
0,0 \%\end{array}$ & & $\begin{array}{l}2 \\
100,0 \%\end{array}$ & & $\begin{array}{l}2 \\
100,0 \%\end{array}$ \\
\hline & 6 & $\begin{array}{l}\text { Total } \\
\%\end{array}$ & $\begin{array}{l}1 \\
33,3 \%\end{array}$ & 0 & $\begin{array}{l}0 \\
0,0 \%\end{array}$ & $\begin{array}{l}0 \\
0,0 \%\end{array}$ & 0 & & 2 (2 $66,7 \%$ & & $\begin{array}{l}3 \\
100,0 \%\end{array}$ \\
\hline & 7 & $\begin{array}{l}\text { Total } \\
\%\end{array}$ & $\begin{array}{l}0 \\
0,0 \%\end{array}$ & $\begin{array}{l}0 \\
0,0 \%\end{array}$ & $\begin{array}{l}0 \\
0,0 \%\end{array}$ & $\begin{array}{l}1 \\
9,1 \%\end{array}$ & $\begin{array}{l}4 \\
36,4 \%\end{array}$ & & $\begin{array}{l}6 \\
54,5 \%\end{array}$ & & $\begin{array}{l}11 \\
100,0 \%\end{array}$ \\
\hline & 8 & $\begin{array}{l}\text { Total } \\
\%\end{array}$ & $\begin{array}{l}3 \\
9,1 \%\end{array}$ & $\begin{array}{l}4 \\
12,1 \%\end{array}$ & 2 & $\begin{array}{l}3 \\
9,1 \%\end{array}$ & $\begin{array}{l}4 \\
12,1 \%\end{array}$ & & $\begin{array}{l}17 \\
51,5 \%\end{array}$ & & $\begin{array}{l}33 \\
100,0 \%\end{array}$ \\
\hline \multicolumn{2}{|l|}{ Total } & $\begin{array}{l}\text { Total } \\
\%\end{array}$ & $\begin{array}{l}4 \\
8,2 \%\end{array}$ & $\begin{array}{l}4 \\
8,2 \%\end{array}$ & $\begin{array}{l}2 \\
4,1 \%\end{array}$ & $\begin{array}{l}4 \\
8,2 \%\end{array}$ & $\begin{array}{l}8 \\
16,3 \%\end{array}$ & & $\begin{array}{l}27 \\
55,1 \%\end{array}$ & & $\begin{array}{l}49 \\
100,0 \%\end{array}$ \\
\hline
\end{tabular}

$X^{2}=11,54, d f=15, p=0,17$

Table 3: Distribution of children according to the number of cigarettes smoked per week in respect of their grade levels.

\begin{tabular}{|c|c|c|c|c|c|c|c|}
\hline & & & \multicolumn{4}{|c|}{ Does tobacco smoke damage the environment or only the smoker } & \multirow[b]{2}{*}{ Total } \\
\hline & & & Yes & No & I don't know & $\begin{array}{l}\text { I have never thought } \\
\text { about it before }\end{array}$ & \\
\hline \multirow{4}{*}{ Class } & 5 & $\begin{array}{c}\text { Total } \\
\%\end{array}$ & $\begin{array}{c}32 \\
91,4 \%\end{array}$ & $\begin{array}{c}0 \\
0,0 \%\end{array}$ & $\begin{array}{c}3 \\
8,6 \%\end{array}$ & $\begin{array}{c}0 \\
0,0 \%\end{array}$ & $\begin{array}{c}35 \\
100,0 \%\end{array}$ \\
\hline & 6 & $\begin{array}{c}\text { Total } \\
\%\end{array}$ & $\begin{array}{c}58 \\
87,9 \%\end{array}$ & $\begin{array}{c}0 \\
0,0 \%\end{array}$ & $\begin{array}{c}5 \\
7,6 \%\end{array}$ & $\begin{array}{c}3 \\
4,5 \%\end{array}$ & $\begin{array}{c}66 \\
100,0 \%\end{array}$ \\
\hline & 7 & $\begin{array}{c}\text { Total } \\
\%\end{array}$ & $\begin{array}{c}38 \\
92,7 \%\end{array}$ & $\begin{array}{c}0 \\
0,0 \%\end{array}$ & $\begin{array}{c}2 \\
4,9 \%\end{array}$ & $\begin{array}{c}1 \\
2,4 \%\end{array}$ & $\begin{array}{c}41 \\
100,0 \%\end{array}$ \\
\hline & 8 & $\begin{array}{c}\text { Total } \\
\%\end{array}$ & $\begin{array}{c}71 \\
93,4 \%\end{array}$ & $\begin{array}{c}1 \\
1,3 \%\end{array}$ & $\begin{array}{c}4 \\
5,3 \%\end{array}$ & $\begin{array}{c}0 \\
0,0 \%\end{array}$ & $\begin{array}{c}76 \\
100,0 \%\end{array}$ \\
\hline \multicolumn{2}{|c|}{ Total } & $\begin{array}{c}\text { Total } \\
\%\end{array}$ & $\begin{array}{c}199 \\
91,3 \%\end{array}$ & $\begin{array}{c}1 \\
0,5 \%\end{array}$ & $\begin{array}{c}14 \\
6,4 \%\end{array}$ & $\begin{array}{c}4 \\
1,8 \%\end{array}$ & $\begin{array}{c}218 \\
100,0 \%\end{array}$ \\
\hline
\end{tabular}

Table 4: Distribution of responses to the question whether tobacco smoke harms the environment or only the smoker in respect of the grade levels.

the attitude of schools towards these matters. The survey was conducted among students of the $7^{\text {th }}, 8^{\text {th }}$ and $9^{\text {th }}$ grades between age 13 and 15 . The results obtained demonstrate that $42.7 \%$ of students have already smoked their first cigarettes; this represents $41.4 \%$ of the boys and $43.3 \%$ of the girls. $9.3 \%$ of the students claimed themselves to be smokers; this corresponds to $9.3 \%$ of the boys and $8.9 \%$ of the girls. Total of $2.1 \%$ claimed to be everyday smokers; this contributes to $2.3 \%$ of the boys and $1.9 \%$ of the girls. In this study if we only take into consideration the 
Citation: Veres KT, Zvezdin B, Vukosav D (2015) Assessment of Knowledge, Behavior and Attitude of School Children towards Smoking. J Pulm Respir Med 5: 297. doi:10.4172/2161-105X.1000297

Page 4 of 7

\begin{tabular}{|c|c|c|c|c|c|c|c|}
\hline & & & \multicolumn{4}{|c|}{ Does tobacco smoke damage the environment or only the smoker } & \multirow[b]{2}{*}{ Total } \\
\hline & & & Yes & No & I don't know & $\begin{array}{l}\text { I have never thought about } \\
\text { it before }\end{array}$ & \\
\hline \multirow{2}{*}{ Gender } & Male & $\begin{array}{c}\text { Total } \\
\%\end{array}$ & $\begin{array}{c}101 \\
89,4 \%\end{array}$ & $\begin{array}{c}0 \\
0,0 \%\end{array}$ & $\begin{array}{c}9 \\
8,0 \%\end{array}$ & $\begin{array}{c}3 \\
2,7 \%\end{array}$ & $\begin{array}{c}113 \\
100,0 \%\end{array}$ \\
\hline & Female & $\begin{array}{c}\text { Total } \\
\%\end{array}$ & $\begin{array}{c}98 \\
93,3 \%\end{array}$ & $\begin{array}{c}1 \\
1,0 \%\end{array}$ & $\begin{array}{c}5 \\
4,8 \%\end{array}$ & $\begin{array}{c}1 \\
1,0 \%\end{array}$ & $\begin{array}{c}105 \\
100,0 \%\end{array}$ \\
\hline \multicolumn{2}{|c|}{ Total } & $\begin{array}{c}\text { Total } \\
\%\end{array}$ & $\begin{array}{c}199 \\
91,3 \%\end{array}$ & $\begin{array}{c}1 \\
0,5 \%\end{array}$ & $\begin{array}{c}14 \\
6,4 \%\end{array}$ & $\begin{array}{c}4 \\
1,8 \%\end{array}$ & $\begin{array}{c}218 \\
100,0 \%\end{array}$ \\
\hline
\end{tabular}

Table 5: Distribution of responses to the question whether tobacco smoke harms the environment or only the smoker in respect of the gender.

\begin{tabular}{|c|c|c|c|c|c|c|c|}
\hline & & & \multicolumn{4}{|c|}{ Does tobacco smoke damage the environment or only the smoker } & \multirow[b]{2}{*}{ Total } \\
\hline & & & Yes & No & I don't know & $\begin{array}{l}\text { I have never thought } \\
\text { about it before }\end{array}$ & \\
\hline \multirow{2}{*}{ Class } & Hungarian & $\begin{array}{c}\text { Total } \\
\%\end{array}$ & $\begin{array}{c}70 \\
86,4 \%\end{array}$ & $\begin{array}{c}0 \\
0,0 \%\end{array}$ & $\begin{array}{c}7 \\
8,6 \%\end{array}$ & $\begin{array}{c}4 \\
4,9 \%\end{array}$ & $\begin{array}{c}81 \\
100,0 \%\end{array}$ \\
\hline & Serbian & $\begin{array}{c}\text { Total } \\
\%\end{array}$ & $\begin{array}{c}129 \\
94,2 \%\end{array}$ & $\begin{array}{c}1 \\
0,7 \%\end{array}$ & $\begin{array}{c}7 \\
5,1 \%\end{array}$ & $\begin{array}{c}0 \\
0,0 \%\end{array}$ & $\begin{array}{c}137 \\
100,0 \%\end{array}$ \\
\hline \multicolumn{2}{|c|}{ Total } & $\begin{array}{c}\text { Total } \\
\%\end{array}$ & $\begin{array}{c}199 \\
91,3 \%\end{array}$ & $\begin{array}{c}1 \\
0,5 \%\end{array}$ & $\begin{array}{c}14 \\
6,4 \%\end{array}$ & $\begin{array}{c}4 \\
1,8 \%\end{array}$ & $\begin{array}{c}218 \\
100,0 \%\end{array}$ \\
\hline \multicolumn{8}{|c|}{$x^{2}=8,68, d f=3, p=0,034$} \\
\hline
\end{tabular}

Table 6: Distribution of responses to the question whether tobacco smoke harms the environment or only the smoker in respect of the nationality of children.

\begin{tabular}{|c|c|c|c|c|c|c|c|c|}
\hline & & & \multicolumn{5}{|c|}{ Second-hand smoking is } & \multirow[b]{2}{*}{ Total } \\
\hline & & & $\begin{array}{l}\text { Smoking } \\
\text { society }\end{array}$ & in Occasional smoking & $\begin{array}{l}\text { Staying in a smoky } \\
\text { room }\end{array}$ & I don't know & $\begin{array}{l}\text { I have never } \\
\text { thought about it }\end{array}$ & \\
\hline \multirow{4}{*}{ Class } & 5th & $\begin{array}{l}\text { Total } \\
\%\end{array}$ & $\begin{array}{l}3 \\
9,1 \%\end{array}$ & $\begin{array}{l}3 \\
9,1 \%\end{array}$ & $\begin{array}{l}8 \\
24,2 \%\end{array}$ & $\begin{array}{l}17 \\
51,5 \%\end{array}$ & 2 & $\begin{array}{l}33 \\
100,0 \%\end{array}$ \\
\hline & 6th & $\begin{array}{l}\text { Total } \\
\%\end{array}$ & $\begin{array}{l}8 \\
12,1 \%\end{array}$ & $\begin{array}{l}0 \\
0,0 \%\end{array}$ & $\begin{array}{l}33 \\
50,0 \%\end{array}$ & $\begin{array}{l}19 \\
28,8 \%\end{array}$ & $\begin{array}{l}6 \\
9,1 \%\end{array}$ & $\begin{array}{l}66 \\
100,0 \%\end{array}$ \\
\hline & 7th & $\begin{array}{l}\text { Total } \\
\%\end{array}$ & $\begin{array}{l}8 \\
19,5 \%\end{array}$ & $\begin{array}{l}2 \\
4,9 \%\end{array}$ & $\begin{array}{l}16 \\
39,0 \%\end{array}$ & $\begin{array}{l}9 \\
22,0 \%\end{array}$ & $\begin{array}{l}6 \\
14,6 \%\end{array}$ & $\begin{array}{l}41 \\
100,0 \%\end{array}$ \\
\hline & 8th & $\begin{array}{l}\text { Total } \\
\%\end{array}$ & $\begin{array}{l}8 \\
10,5 \%\end{array}$ & $\begin{array}{l}9 \\
11,8 \%\end{array}$ & $\begin{array}{l}40 \\
52,6 \%\end{array}$ & $\begin{array}{l}14 \\
18,4 \%\end{array}$ & $\begin{array}{l}5 \\
6,6 \%\end{array}$ & $\begin{array}{l}76 \\
100,0 \%\end{array}$ \\
\hline \multicolumn{2}{|l|}{ Total } & $\begin{array}{l}\text { Total } \\
\%\end{array}$ & $\begin{array}{l}27 \\
12,5 \%\end{array}$ & $\begin{array}{l}14 \\
6,5 \%\end{array}$ & $\begin{array}{l}97 \\
44,9 \%\end{array}$ & $\begin{array}{l}59 \\
27,3 \%\end{array}$ & $\begin{array}{l}19 \\
8,8 \%\end{array}$ & $\begin{array}{l}216 \\
100,0 \%\end{array}$ \\
\hline
\end{tabular}

Table 7: Distribution of responses to the question what second-hand smoking is in respect of grade levels.

\begin{tabular}{|c|c|c|c|c|c|c|c|c|}
\hline & & & \multicolumn{5}{|c|}{ Second-hand smoking is } & \multirow[b]{2}{*}{ Total } \\
\hline & & & $\begin{array}{l}\text { Smoking in } \\
\text { company }\end{array}$ & $\begin{array}{c}\text { Occasional } \\
\text { smoking }\end{array}$ & $\begin{array}{c}\text { Staying in a smoky } \\
\text { room }\end{array}$ & I don't know & $\begin{array}{l}\text { I have never } \\
\text { thought about it }\end{array}$ & \\
\hline \multirow{2}{*}{ Gender } & Male & $\begin{array}{c}\text { Total } \\
\%\end{array}$ & $\begin{array}{c}13 \\
11,6 \%\end{array}$ & $\begin{array}{c}6 \\
5,4 \%\end{array}$ & $\begin{array}{c}46 \\
41,1 \%\end{array}$ & $\begin{array}{c}31 \\
27,7 \%\end{array}$ & $\begin{array}{c}16 \\
14,3 \%\end{array}$ & $\begin{array}{c}112 \\
100,0 \%\end{array}$ \\
\hline & Female & $\begin{array}{c}\text { Total } \\
\%\end{array}$ & $\begin{array}{c}14 \\
13,5 \%\end{array}$ & $\begin{array}{c}8 \\
7,7 \%\end{array}$ & $\begin{array}{c}51 \\
49,0 \%\end{array}$ & $\begin{array}{c}28 \\
26,9 \%\end{array}$ & $\begin{array}{c}3 \\
2,9 \%\end{array}$ & $\begin{array}{c}104 \\
100,0 \%\end{array}$ \\
\hline \multicolumn{2}{|c|}{ Total } & $\begin{array}{c}\text { Total } \\
\%\end{array}$ & $\begin{array}{c}27 \\
12,5 \%\end{array}$ & $\begin{array}{c}14 \\
6,5 \%\end{array}$ & $\begin{array}{c}97 \\
44,9 \%\end{array}$ & $\begin{array}{c}59 \\
27,3 \%\end{array}$ & $\begin{array}{c}19 \\
8,8 \%\end{array}$ & $\begin{array}{c}216 \\
100,0 \%\end{array}$ \\
\hline \multicolumn{9}{|c|}{$x^{2}=9,34, d f=4, p=0,051$} \\
\hline
\end{tabular}

Table 8: Distribution of responses to the question what second-hand smoking is in respect of gender. 
Citation: Veres KT, Zvezdin B, Vukosav D (2015) Assessment of Knowledge, Behavior and Attitude of School Children towards Smoking. J Pulm Respir Med 5: 297. doi:10.4172/2161-105X.1000297

Page 5 of 7

\begin{tabular}{|c|c|c|c|c|c|c|c|c|}
\hline & & & \multicolumn{5}{|c|}{ Second-hand smoking is } & \multirow{2}{*}{ Total } \\
\hline & & & $\begin{array}{l}\text { Smoking in } \\
\text { company }\end{array}$ & $\begin{array}{l}\text { Occasional } \\
\text { smoking }\end{array}$ & $\begin{array}{l}\text { Staying in a } \\
\text { smoky room }\end{array}$ & I don't know & $\begin{array}{l}\text { I have never } \\
\text { thought about it }\end{array}$ & \\
\hline \multirow{2}{*}{ Class } & Hungarian & $\begin{array}{c}\text { Total } \\
\%\end{array}$ & $\begin{array}{c}11 \\
13,6 \%\end{array}$ & $\begin{array}{c}3 \\
3,7 \%\end{array}$ & $\begin{array}{c}12 \\
14,8 \%\end{array}$ & $\begin{array}{c}43 \\
53,1 \%\end{array}$ & $\begin{array}{c}12 \\
14,8 \%\end{array}$ & $\begin{array}{c}81 \\
100,0 \%\end{array}$ \\
\hline & Serbian & $\begin{array}{c}\text { Total } \\
\%\end{array}$ & $\begin{array}{c}16 \\
11,9 \%\end{array}$ & $\begin{array}{c}11 \\
8,1 \%\end{array}$ & $\begin{array}{c}85 \\
63,0 \%\end{array}$ & $\begin{array}{c}16 \\
11,9 \%\end{array}$ & $\begin{array}{c}7 \\
5,2 \%\end{array}$ & $\begin{array}{c}135 \\
100,0 \%\end{array}$ \\
\hline \multicolumn{2}{|c|}{ Total } & $\begin{array}{c}\text { Total } \\
\%\end{array}$ & $\begin{array}{c}27 \\
12,5 \%\end{array}$ & $\begin{array}{c}14 \\
6,5 \%\end{array}$ & $\begin{array}{c}97 \\
44,9 \%\end{array}$ & $\begin{array}{c}59 \\
27,3 \%\end{array}$ & $\begin{array}{c}19 \\
8,8 \%\end{array}$ & $\begin{array}{c}216 \\
100,0 \%\end{array}$ \\
\hline \multicolumn{9}{|c|}{$x^{2}=64,64, d f=4, p=0,00$} \\
\hline
\end{tabular}

Table 9: Distribution of responses to the question what second-hand smoking is in respect of nationality

\begin{tabular}{|c|c|c|c|c|c|}
\hline & & & \multicolumn{2}{|c|}{ Have you ever tried smoking } & \multirow{2}{*}{ Total } \\
\hline & & & Yes & No & \\
\hline \multirow{3}{*}{$\begin{array}{c}\text { Do you allow guests to smoke in } \\
\text { your house }\end{array}$} & Yes & $\begin{array}{c}\text { Total } \\
\%\end{array}$ & $\begin{array}{c}67 \\
41,6 \%\end{array}$ & $\begin{array}{c}94 \\
58,4 \%\end{array}$ & $\begin{array}{c}161 \\
100,0 \%\end{array}$ \\
\hline & No & $\begin{array}{c}\text { Total } \\
\%\end{array}$ & $\begin{array}{c}6 \\
24,0 \%\end{array}$ & $\begin{array}{c}19 \\
76,0 \%\end{array}$ & $\begin{array}{c}25 \\
100,0 \%\end{array}$ \\
\hline & I don't know & $\begin{array}{c}\text { Total } \\
\%\end{array}$ & $\begin{array}{c}0 \\
0,0 \%\end{array}$ & $\begin{array}{c}6 \\
100,0 \%\end{array}$ & $\begin{array}{c}6 \\
100,0 \%\end{array}$ \\
\hline \multicolumn{2}{|l|}{ Total } & $\begin{array}{c}\text { Total } \\
\%\end{array}$ & $\begin{array}{c}73 \\
38,0 \%\end{array}$ & $\begin{array}{c}119 \\
62,0 \%\end{array}$ & $\begin{array}{c}192 \\
100,0 \%\end{array}$ \\
\hline \multicolumn{6}{|c|}{$x^{2}=6,64, d f=2, p=0,036$} \\
\hline
\end{tabular}

Table 10: Occurrence of trying smoking depending on whether the guests are allowed to smoke in the house or not.

\begin{tabular}{|c|c|c|c|c|c|}
\hline & & & \multicolumn{2}{|c|}{ Have you ever tried smoking } & \multirow{2}{*}{ Total } \\
\hline & & & Yes & No & \\
\hline \multirow{3}{*}{$\begin{array}{l}\text { Have any of your friends smoked in your } \\
\text { presence }\end{array}$} & Yes & $\begin{array}{c}\text { Total } \\
\%\end{array}$ & $\begin{array}{c}64 \\
50,4 \%\end{array}$ & $\begin{array}{c}63 \\
49,6 \% \\
\end{array}$ & $\begin{array}{c}127 \\
100,0 \%\end{array}$ \\
\hline & No & $\begin{array}{c}\text { Total } \\
\%\end{array}$ & $\begin{array}{c}7 \\
11,5 \%\end{array}$ & $\begin{array}{c}54 \\
88,5 \%\end{array}$ & $\begin{array}{c}61 \\
100,0 \%\end{array}$ \\
\hline & I don't know & $\begin{array}{c}\text { Total } \\
\%\end{array}$ & $\begin{array}{c}5 \\
25,0 \%\end{array}$ & $\begin{array}{c}15 \\
75,0 \%\end{array}$ & $\begin{array}{c}20 \\
100,0 \%\end{array}$ \\
\hline \multicolumn{2}{|l|}{ Total } & $\begin{array}{c}\text { Total } \\
\%\end{array}$ & $\begin{array}{c}76 \\
36,5 \%\end{array}$ & $\begin{array}{c}132 \\
63,5 \%\end{array}$ & $\begin{array}{c}208 \\
100,0 \%\end{array}$ \\
\hline
\end{tabular}

Table 11: Occurrence of trying smoking depending on whether the peers are smoking or not in the student's presence.

students between age 13 and 15 of $6^{\text {th }}, 7^{\text {th }}$ and $8^{\text {th }}$ grades, the percentage of students who smoked their first cigarettes among boys is $40.81 \%$ and $35.6 \%$ among girls. The occurrence of everyday smokers in the studied age group was $7 \%$, which represents $10 \%$ of the boys and $3 \%$ of the girls. In a survey that was conducted in the primary schools in the area of Novi Sad in 2003, the occurrence of everyday smokers amongst students between age 13 and 15 is $9 \%$, that was $7.95 \%$ of the girls and $7.51 \%$ of the boys [7].

According to these statistics in all Serbia a higher percentage of girls than boys tried cigarettes in the studied age group which supports the trend in the world. Smoking on a regular basis (every week) is more likely among girls than among boys in 19 of 31 European countries $[8,9]$. This difference between the genders which varies from $1 \%$ to $11 \%$ is most prevalent in Great Britain, the country that has the longest history of smoking. In 2002 in Scotland 24\% of the fifteen-year-old girls were regular smokers (every week) compared to the boys where the percentage was $16 \%$. Furthermore, from 1994 the rate of smokers among boys has significantly dropped from $20 \%$, however, this trend among girls is not noticeable [10]. In our research, however, the percentage of those who have already smoked their first cigarettes was lower than the national average, and in respect of gender the results 
show that smoking among boys is more likely than among girls which differ from the national trend. Concerning everyday smokers this difference is significant and perhaps this could be explained by the fact that the study was conducted in an underdeveloped municipality.

In most Western countries starting smoking is most frequent among the adolescent (as opposed to among younger children). In 2009, In Netherlands 7\% of the eleven-year-old have already smoked their first cigarettes. This percentage increases to $45 \%$ among the fourteen-year-old and to $62 \%$ among the seventeen-year-old [11]. This rate and trend of smoking is similar to those in Great Britain [12], USA [13] and New Zealand [14]. In this survey, the percentage is $17.1 \%$ among the eleven-year-old, which rises to $46.3 \%$ at the age of 14 and to $54.5 \%$ at the age of 15 . The above-mentioned countries are considered to be developed countries with a stricter control over tobacco use and advertising tobacco products, they prohibited the sale of tobacco products to young people and smoking in public places, additionally, they carried out a variety of other measures achieving a decrease in tobacco use, and therefore these differences in the statistics are not surprising. In Australia from 2011 onwards there has been a $50 \%$ decline in the use of tobacco products among the adolescent, however, currently on average $10 \%$ of the adolescent between age 14 to 19 consume tobacco products on daily or weekly basis or more rarely [15].

From the above statistics it can be clearly seen that among the students of primary schools, especially in Serbia where smoking in these ages remains popular, stopping the youth from "experimenting" with cigarettes or getting them to quit smoking would play a major role in preventing the troubles of nicotine addiction and the diseases it triggers. Every aspect of the addiction can be considered as a highly complex matter during which the function of the nervous processes that are responsible for controlling the behaviour associated with the object of an addiction is changed by means of consuming the object of addiction [16].

There is a wide range of factors that affect whether young people will start and continue smoking. It depends on the nature of each child, depends on their closer environment, as well as in the wider social environment $[17,18]$. Some studies conducted in the USA have pointed out the differences between the genders in respect of these factors. It showed the differences in the schoolchildren's perception of their bodies (girls are more concerned about their appearance and they believe that they can control the body weight by smoking cigarettes), influence of control (girls are more likely to smoke cigarettes in order to get rid of negative emotions), self-esteem (low self-esteem has a greater impact on girls) [19]. In this study $60 \%$ of tested students said that their friends smoked in their presence, $47 \%$ of them said that tobacco smoke bothered them, yet $71.4 \%$ remained in the company of smokers even though the smoker did not put out the cigarette thus disregarding the fact that his/her friend (the participant in the test) expressed previously that they had disliked the cigarette smoke. The quantitative studies confirmed that it is more likely for boys or girls to start smoking if their friends are already smokers [20]. In this study we showed that peers who smoked in a student's presence does have an effect on whether the student tried smoking or not. About $50 \%$ of those students whose peers smoked in their presence had tried cigarettes, while $88.50 \%$ of the students in whose presence their peers never smoked have never tried cigarettes.

A longitudinal study was conducted in the USA on 214 parents and 314 descendants, who were at least 11 years old. This study proved that children of smoking parents in $29 \%$ of the cases and children of former smokers in $23 \%$ of cases smoked in the previous year, however, among the children of parents who are non-smokers the occurrence of smoking in the previous year is only $8 \%$ [21]. In our study among children whose parents were non-smokers only $26.4 \%$ tried cigarettes compared to $51 \%$ of those whose both parents smokes, $40 \%$ of those whose only father smoke and $31 \%$ of those whose only mother smoke. At the same time, children of non-smoking parents in $16.7 \%$ of cases were everyday smokers compared to $41.2 \%$ of children whose both parents smoked, $27.3 \%$ of those whose fathers were the only smokers and $12.5 \%$ of those whose mothers were the only smokers. As much as $74.5 \%$ of children were exposed to second-hand smoking in their homes. Leonardi-Bee and his associates performed a meta-analysis by which they concluded that parental smoking is a significant risk factor in the children's future attitude towards the tobacco [22].

Prevalence of smoking among young people in Germany decreased to $12 \%$ in 2012 as opposed to this percentage being $28 \%$ in 2001 . In Germany several measures have been carried out recently to control the tobacco use. The most successful measures in the prevention of smoking among the young people were those which were implemented at the level of the entire population. The most efficient among these are the significantly increased taxes and the smoke-free legislation (German Non-Smokers' Protection Act). On the other hand, health education and the training of young people in schools have had a marginal impact too on the youth smoking prevention [23].

In this study, the number of students who smoked their first cigarettes is slightly lower than the statel average, the number of everyday smokers is much higher than the state average. We must point out several limitations of the study. First of all, the relatively small number of respondents, although the response of schoolchildren was satisfactory and there were no incorrectly filled out questionnaires. Secondly, the questionnaire was self-reported and not validated, so there is the possibility that the prevalence of smokers is higher than shown. The frequency of regular smokers on a daily or weekly basis among the tested students is significantly higher than the state average but it does not differ from the results in Croatia, Hungary, Slovakia and Slovenia [6]. Two-thirds of the interviewed children come from families where at least one parent is a smoker, and three-quarter of them are exposed to second-hand smoking in their homes - this does not deviate from the national average nor from the average in Montenegro, Croatia, Bosnia and Herzegovina, but it is a much higher rate than it is in Hungary, Romania and Slovenia [6].

\section{Conclusions}

In this study it has been shown that a large percentage of surveyed school children between 11 and 15 years old have tried cigarettes, and the number of daily smokers is worryingly high. Three-quarters of the children are exposed to second-hand smoking, and nearly half of them do not know what it is means. The results of the study clearly indicate the need for primary prevention of cigarette smoking as an important part of health education to children in order for them to acquire the appropriate skills to resist peer pressure and refuse to smoke so they can make their own decision not to smoke and to understand the consequences of smoking. At the same time, smoke-free environment should be ensured for children and joint education of children and parents would be very necessary because the parents themselves may not be aware of the negative impact tobacco has on the physical and mental development of their children.

\section{References}

1. Mackay J, Eriksen M, Shafey O (2012) The Tobacco Atlas. Atlanta: American 
Citation: Veres KT, Zvezdin B, Vukosav D (2015) Assessment of Knowledge, Behavior and Attitude of School Children towards Smoking. J Pulm Respir Med 5: 297. doi:10.4172/2161-105X.1000297

Cancer Society.

2. Warren CW, Jones NR, Eriksen MP, Asma S; Global Tobacco Surveillance System (GTSS) collaborative group (2006) Patterns of global tobacco use in young people and implications for future chronic disease burden in adults. Lancet 367: 749-753.

3. Hamilton WL, Turner-Bowker DM, Celebucki CC, Connolly GN (2002) Cigarette advertising in magazines: the tobacco industry response to the Master Settlement Agreement and to public pressure. Tob Control 11 Suppl 2: ii54-58.

4. Patnode CD, O'Connor E, Whitlock EP, Perdue LA, Soh C, et al. (2013) Primary Care-Relevant Interventions for Tobacco Use Prevention and Cessation in Children and Adolescents: A Systematic Evidence Review for the U.S. Preventive Services Task Force. Ann Intern Med 158: 253-260.

5. Vardavas C (2010) Key points in preventing tobacco use among adolescents Tob Induc Dis 8: 1.

6. Baeza-Loya S, Viswanath H, Carter A, Molfese DL, Velasquez KM, et al. (2014) Perceptions about e-cigarette safety may lead to e-smoking during pregnancy. Bull Menninger Clin 78: 243-252.

7. Zvezdin B, Kojicic M, Kopcanski M, Loncarevic M (2003) The smoking habit frequency among primary and secondary school pupils in the area of Novi Sad. ERS Annual Congress, Abstract book, Vienna.

8. Currie C, Roberts C, Morgan A (2004) Health Behaviour in School-aged Children Study: International Report from the 2001/2002 Survey. Young People's Health in Context 4: 63-73.

9. Alexander L, Currie C, Todd J, Smith R (2004) How are Scotland's young people doing? A cross-national perspective on health risk. HBSC Briefing Paper: 6.

10. Currie C, Fairgrieve J, Akhtar P (2003) Smoking, Drinking and Drug Use Among 13 And 15 Year Olds in Scotland In 2002. Scottish Schools Adolescent Lifestyle and Substance Use Survey (SALSUS) National Report. TSO: 17-79.

11. Hiemstra M, Otten R, Engels RC (2012) Smoking onset and the time-varying effects of self-efficacy, environmental smoking, and smoking-specific parenting by using discrete-time survival analysis. J Behav Med 35: 240-251.

12. Bridges S, Gill V, Omole T, Sutton R, Wright V (2011) Smoking, drinking and drug use among young people in England in 2010. NHS: 17-70.

13. Eaton DK, Kann L, Kinchen S, Shanklin S, Ross J, et al. (2010) Youth risk behavior surveillance - United States, 2009. MMWR Surveill Summ 59: 1-142.

14. Paynter J, Edwards R, Schluter PJ, McDuff I (2009) Point of sale tobacco displays and smoking among 14-15 year olds in New Zealand: a cross-sectiona study. Tob Control 18: 268-274.

15. Hemphill SA, Heerde JA, Herrenkohl TI, Toumbourou JW, Catalano RF (2012) The impact of school suspension on student tobacco use: a longitudinal study in Victoria, Australia, and Washington State, United States. Health Educ Behav 39: $45-56$.

16. Hinic D, Mihajlovic G, Spiric Z, Dukic-Dejanovic S, Jovanovic M (2008) Excessive Internet use--addiction disorder or not? Vojnosanit Pregl 65: 763767.

17. Pierce JP, Distefan JM, Hill D (2004) Adolescent smoking. Tobacco and Public Health: Science and Policy. Oxford: Oxford University Press: 315-328.

18. Kobus K (2003) Peers and adolescent smoking. Addiction 98 Suppl 1: 37-55.

19. Lambert M, Hublet A, Verduyckt (2002) Gender Differences in Smoking in Young People. Belgium: Flemish Institute for Health Promotion: 1-29.

20. Avenevoli S, Merikangas KR (2003) Familial influences on adolescent smoking Addiction 98 Suppl 1: 1-20.

21. Vuolo M, Staff J (2013) Parent and child cigarette use: a longitudinal, multigenerational study. Pediatrics 132: e568-577.

22. Leonardi-Bee J, Jere ML, Britton J (2011) Exposure to parental and sibling smoking and the risk of smoking uptake in childhood and adolescence: a systematic review and meta-analysis. Thorax 66: 847-855.

23. Schaller K, Braun S, Viarisio V, Pötschke-Langer M, Hanewinkel R (2014) German Cancer Research Center. (edn) Tobacco prevention in Germany-what works? From Science to Politics: 1-7. 